\title{
Optimizing Parkinson's disease Diagnosis: A Clinical Review Magnetic Resonance Imaging (MRI)
}

Jodi Krustesen 1*, David Chris 1

Department of Neuroscience, Iran

* Corresponding Author: Jodi Krustesen, Department of NeuroScience, Iran

Received date: April 25,2018 ;Accepted date : May 15,2018; Published date: June 042018.

Citation: Jodi Krustesen, David Chris Optimizing Parkinson's disease Diagnosis: A Clinical Review Magnetic Resonance Imaging (MRI).

Doi: 10.31579/2578-8868/031

Copyright : (c) 2018 Jodi Krustesen. This is an open-access article distributed under the terms of The Creative Commons Attribution License, which permits unrestricted use, distribution, and reproduction in any medium, provided the original author and source are credited.

\begin{abstract}
The diagnosis of Parkinson's disease (PD) currently relies almost exclusively on the clinical judgment of an experienced neurologist, ideally a specialist in movement disorders. However, such clinical diagnosis is often incorrect in a large percentage of patients, particularly in the early stages of the disease. A commercially available, objective and quantitative marker of nigrostriatal neurodegeneration was recently provided by 123-iodine ${ }^{123}$ I-ioflupane SPECT imaging, which is however unable to differentiate PD from a variety of other parkinsonian syndromes associated with striatal dopamine deficiency. There is evidence to support an algorithm utilizing a dual neuroimaging strategy combining ${ }^{123}$ Iioflupane SPECT and the noradrenergic receptor ligand ${ }^{123}$ I-metaiodobenzylguanidine (MIBG), which assesses the post-ganglion peripheral autonomic nervous system. Evolving concepts regarding the synucleinopathy affecting the central and peripheral autonomic nervous systems as part of a multisystem disease are reviewed to sustain such strategy.
\end{abstract}

\section{Introduction}

Parkinson's disease (PD) is the second most common neurodegenerative disorder of aging with a prevalence of about $1-2 \%$ in adults over age 60 in industrialized countries. ${ }^{1}$ In excess of one million people suffer from PD in the United States alone and, due to the progressive aging of the population, the importance of PD as a public health issue is expected to increase. The incidence of the disease is increasing over time, particularly in males, and it is projected that by 2030 the number of individuals with PD in the world will triple. In the United States, annual medical costs associated with PD, including doctor visits, medications, physical and speech therapies, and comorbidities of the disease (such as depression and dementia) are significant burdens to the healthcare system, with a total cost to the nation projected to be $\$ 23$ billion annually.

The burden of disease is likely even greater considering the widespread presence of Lewy bodies in PD and the multifaceted symptoms and signs associated with this syndrome. ${ }^{5}$ Put another way, our traditional definition of parkinsonism is just the -tip of the iceberg\| of what PD truly appears to be.And if Lewy bodies remain a cardinal pathologic feature of PD, it is now clear that PD is just one aspect of a larger synucleinopathy, best described as multisystem Lewy body disease (MLBD) and comprising PD, pure autonomic failure (PAF), and dementia with Lewy bodies (DLB).

These evolving concepts regarding the widespread nature of PD synucleinopathy has also led to challenges in diagnosis, which, as typically defined, relies on the clinical acumen of an experienced neurologist, ideally one who is specialized in movement disorders. For decades, diagnosis has been based on the recognition of four cardinal signs: resting tremor, bradykinesia, rigidity and postural instability. However, recent evidence has shown that the clinical diagnosis is often incorrect in an alarming percentage of patients, particularly in the early stages of the disease. Obviously, this could affect day-to-day patient care and seriously confound the interpretation of clinical trials. In this paper, we provide supportive evidence for a dual imaging algorithm that could markedly improve the diagnostic accuracy of MLBD, including its dominant clinical manifestation-idiopathic PD.
This brief review focuses on magnetic resonance imaging (MRI) of Parkinson's disease (PD), which is the second most common neurodegenerative disease after Alzheimer's disease (AD). Approximately $1 \%$ of those over 65 years of age have PD. While MRI is presently not able to directly image dopaminergic neuronal loss that underlies PD, it can provide complementary data to that obtained with nuclear tracer imaging. This article will review commonly available and research MRI methods that may provide an imaging measure of disease.

\section{$\mathrm{T}_{\mathbf{2}}$ and $\mathrm{T}_{\mathbf{2}}{ }^{*}$ Imaging}

In the 1980s MRI imaging was first applied in PD, when several groups focused on demonstrating the presence of increased iron in the substantia nigra of individuals with PD. This was followed by Gorell et al. in 1995 who utilized $\mathrm{T}_{2}$ and $\mathrm{T}_{2}{ }^{*}$ imaging of the substantia nigra and showed a separation between those with PD from control participants by using a change in relaxation time constants as a surrogate for increased iron in PD . The focus on $\mathrm{T}_{2}{ }^{*}$ or its reciprocal $\mathrm{R}_{2}{ }^{*}$ has remained an important aspect of nigral imaging protocols, and an excellent demonstration of macroscopic nigral changes attributable to iron was shown by Cho et al. in their 7 Tesla (T) imaging study . One crucial assumption about -ironll based imaging is that the methods reflect upon non-heme iron as opposed to heme-iron, and that while the small pool of free labile iron may be pathogenic - imaging methods are presently sensitive to the more prevalent bound iron that is stored as ferritin or neuromelanin . Today MRI cannot determine if these iron changes arise from neuromelanin in dopaminergic neurons or ferritin in glia or neurons.

\section{Susceptibility-weighted Imaging}

SWI methods exploit the differences in magnetic susceptibility between tissues, and are available on clinical MRI platforms. Using gradient echo (GRE) pulse sequences with long echo time (TE), SWI provides enhanced image contrast for detecting susceptibility variations when combining magnitude and phase data. Specifically, the local field variations are the source of local phase differences in the MRI signal. Phase variations contain both microscopic and macroscopic effects. The phase variations due to microscopic effects mainly originate from local iron deposits whereas the macroscopic effects can be attributed to geometry effects or air/tissue interfaces. 
Complicated tissue geometries such as capillary beds, interstitial spaces, large and small vessels, etc., distort the local field homogeneity and thus induce signal variations. In fact, induced susceptibility differences depend not only on the geometry of such structures, but also on their orientations with respect to the external magnetic field.

\section{Magnetization Transfer Imaging (MTI)}

Magnetization transfer imaging (MTI) utilizes the transfer of magnetization between free water protons and protons associated with macromolecules which provides information about tissue integrity [. The detection of the magnetization transfer (MT) effect in clinical practice is usually limited to the measurement of MT ratios (MTRs), i.e. ratios of signal intensity measured with and without the off resonance saturation pulse. One group has shown the utility of MTR in PD while others have shown its value in atypical parkinsonian conditions.

\section{Resting-state MRI}

The focus of resting-state MRI is on brain activity that occurs in the absence of externally triggered activity. Even in a -resting statell there are physiological variations in brain activity and accompanying blood flow alterations that manifest as fluctuations in the MRI blood oxygen level dependent (BOLD) signal. Spontaneous correlations in BOLD signal can be utilized to determine the -functional connectivityll between different regions.

\section{Clinical Applications}

MRI methods are making their way into the clinic by aiding the neurosurgeon in planning deep brain stimulation (DBS) surgery. Secondly, multi-modality approaches may increase sensitivity to disease states, as shown for example in the combination of structural and iron sensitive imaging. It is hoped that cross-sectional and longitudinal studies will provide insights about the ability of such methods to provide a correlate to disease severity and progression.

\section{References}

1. Tanner CM, Goldman SM. Epidemiology of Parkinson's disease. Neurol Clin. 1996;14:317-335. khttps://doi.org/10.1016/S1474-4422(06)70471-9

2. Rutledge JN, Hilal SK, Silver AJ, Defendini R, Fahn S. Study of movement disorders and brain iron by MR. AJR Am J Roentgenol. 1987;149:365-379.

3. Gorell JM, Ordidge RJ, Brown GG, Deniau JC, Buderer NM, et al. Increased iron-related MRI contrast in the substantia nigra in Parkinson's disease. Neurology. 1995;45:1138-1143. DOI: https://doi.org/10.1212/ WNL. 45.6.1138
Cho ZH, Oh SH, Kim JM, Park SY, Kwon DH, et al. Direct visualization of Parkinson's disease by in vivo human brain imaging using 7.0T magnetic resonance imaging. Mov Disord. 2011;26:713718.https://doi.org/10.1002/mds. 23465

5. Zecca L, Youdim MB, Riederer P, Connor JR, Crichton RR. Iron, brain ageing and neurodegenerative disorders. Nat Rev Neurosci. 2004;5:863-873.

6. Zecca L, Wilms H, Geick S, Claasen JH, Brandenburg LO, et al. Human neuromelanin induces neuroinflammation and neurodegeneration in the rat substantia nigra: implications for Parkinson's disease. Acta Neuropathol. 2008;116:47-55.

7. Jin L, Wang J, Zhao L, Jin H, Fei G, et al. Decreased serum ceruloplasmin levels characteristically aggravate nigral iron deposition in Parkinson's disease. Brain. 2011;134:50 58.https://doi.org/10.1093/brain/awq319

8. Michaeli S, Oz G, Sorce DJ, Garwood M, Ugurbil K, et al. Assessment of brain iron and neuronal integrity in patients with Parkinson's disease using novel MRI contrasts. Mov Disord. 2007;22:334-340.https://doi.org/10.1002/mds.21227

9. Nestrasil I, Michaeli S, Liimatainen T, Rydeen CE, Kotz CM, et al. T1rho and T2rho MRI in the evaluation of Parkinson's disease. J Neurol. 2010;257:964-968.

10. Wang Y, Butros SR, Shuai X, Dai Y, Chen C, et al. Different irondeposition patterns of multiple system atrophy with predominant parkinsonism and idiopathetic Parkinson diseases demonstrated by phase-corrected susceptibility-weighted imaging. AJNR Am J Neuroradiol. 2012;33:266-273DOI: https://doi.org/10.3174/ajnr. A2765.

11. Manova ES, Habib CA, Boikov AS, Ayaz M, Khan A, et al. Characterizing the mesencephalon using susceptibility-weighted imaging. AJNR Am J Neuroradiol. 2009;30:569-574.DOI: https://doi.org/10.3174/ajnr.A1401

12. Gupta D, Saini J, Kesavadas C, Sarma PS, Kishore A. Utility of susceptibility-weighted MRI in differentiating Parkinson's disease and atypical parkinsonism. Neuroradiology. 2010;52:1087-1094.

4. 